\title{
The N-terminal 33 amino acid domain of Siva-1 is sufficient for nuclear localization
}

\author{
J.Y. Chen ${ }^{1}$, L.X. Yang ${ }^{1}$ and Z.F. Huang ${ }^{1,2,3}$ \\ ${ }^{1}$ Institute of Human Virology, Zhongshan School of Medicine, Sun Yat-sen University, Guangzhou, China \\ ${ }^{2}$ Department of Biochemistry, Zhongshan School of Medicine, Sun Yat-sen University, Guangzhou, China \\ ${ }^{3}$ Key Laboratory of Tropical Diseases Control, Sun Yat-sen University, Ministry of Education in China, Guangzhou, China
}

\begin{abstract}
Siva-1 induces apoptosis in multiple pathological processes and plays an important role in the suppression of tumor metastasis, protein degradation, and other functions. Although many studies have demonstrated that Siva-1 functions in the cytoplasm, a few have found that Siva- 1 can relocate to the nucleus. In this study, we found that the first 33 amino acid residues of Siva-1 are required for its nuclear localization. Further study demonstrated that the green fluorescent protein can be imported into the nucleus after fusion with these 33 amino acid residues. Other Siva-1 regions and domains showed less effect on Siva-1 nuclear localization. By site-mutagenesis of all of these 33 amino acid residues, we found that mutants of the first 1-18 amino acids affected Siva-1 nuclear compartmentalization but could not complete this localization independently. In summary, we demonstrated that the N-terminal 33 amino acid residues were sufficient for Siva-1 nuclear localization, but the mechanism of this translocation needs additional investigation.
\end{abstract}

Key words: Siva-1; Nuclear localization signal (NLS); Subcellular compartmentalization; Nuclear localization

\section{Introduction}

Siva-1 was originally identified as a CD27-binding protein with the yeast two-hybrid system (1), and was found to induce apoptosis in multiple pathological processes. Siva-1 consists of several distinct domains: an N-terminal region, a putative amphipathic helical $(\mathrm{SAH})$ region, a death domain homology region (DDHR), and a C-terminal cysteine-rich region that includes a B-BOX domain and a zinc-finger-like domain $(2,3)$. Each of these domains contributes to Siva-1's functions and to its ability to bind to many interactive proteins. In addition to CD27, Siva-1 can interact with the peroxisomal membrane protein (PMP22) (4), tyrosine kinase 2 (Tyk2) (5), pyrin (6), lysophosphatidic acid receptor 2 (LPA2) (7), the CVB3 capsid protein VP2 (8), and CD4 (9). The SAH region, which includes portions of the N-terminal and the DDHR, is responsible for binding to antiapoptotic factor Bcl-xL (10). Recent studies have shown that both tumor suppressor p53 and XIAP, a potent inhibitor of apoptosis, bind to the Siva-1 Nterminal and DDHR regions $(11,12)$. However, no study has demonstrated that Siva-1 can bind to any proteins related to nuclear transportation.
Siva-1 was once considered to be a cytoplasmic protein because of its function in apoptosis (10). However, subsequent studies demonstrated that Siva-1 could accumulate in the nucleus $(6,7)$ and that amino acid (AA) residues 1-47 of the human Siva-1 N-terminal region participate in this nuclear compartmentalization (2). However, no typical nuclear localization signal (NLS) could be found in the Siva-1 protein sequence, and no possible mechanism explaining its nuclear compartmentalization has been described. Defining the mechanism of Siva-1 translocation into the nucleus may facilitate understanding of Siva-1 functions, especially its unknown functions in the nucleus. The aim of the present study was to isolate and identify the NLS region of Siva-1, and to investigate all elements that could be responsible for Siva1 nuclear localization. By deleting different domains and performing site-directed mutagenesis on AA residues, we constructed a series of green fluorescent protein (GFP)Siva-1 mutation fusion proteins. The results demonstrated that a 33 AA sequence of the N-terminal region of Siva-1 was sufficient for transport into the nucleus, and that other domains were less involved in Siva-1 nuclear localization.

Correspondence: Z.F. Huang, N1311 Rm, No. 10 Bld, 74, Zhongshan 2nd Rd, Guangzhou 510080, China. Fax: +86-20-873-32588. E-mail: hzhaof@mail.sysu.edu.cn 
The demonstration that the Siva- $1 \mathrm{~N}$-terminal region is required for nuclear localization should facilitate future efforts to identify the mechanism of Siva-1 nuclear localization and function.

\section{Material and Methods}

\section{Plasmid construction}

Vectors for expression of the murine wild-type Siva-1 and domain deletion and site-directed Siva-1 mutants fused to GFP were constructed by polymerase chain reaction $(P C R)$, and amplified fragments were inserted between the EcoRI and BamHI sites of pLVX-Tight-Puro, a tetracycline-inducible vector (Clontech, USA).

Siva- 1 was used as a template to generate the Siva-1 deletion mutants Siva1-18, Siva1-33, Siva $\Delta 1-33$, Siva $\Delta 114-175$, and Siva $\Delta 142-175$. These PCR products were cloned into the EcoRI and BamHI sites of the pLVXTight-Puro vector.

Three interstitial deletion mutants: Siva $\Delta 36-55$, Siva $\Delta 48-114$, and Siva $\Delta 114-142$, and two site-directed mutants: Siva (Y34F, Y140F) and Siva (Y53F, Y158F) were created using a two-round PCR procedure. Briefly, two separate DNA fragments were generated in a first PCR. These fragments were purified, combined, and reamplified to produce a final PCR product, which was then cloned into BamHI and EcoRI restriction sites. Cysteine mutants were made by gene synthesis (Jierui Biotech, Co., China) and then cloned into BamHI and EcoRI restriction sites of the the pLVX-Tight-Puro vector.

Five additional mutants, Siva mutant1-6, Siva mutant712, Siva mutant13-18, Siva mutant19-24, Siva mutant 2533, were constructed by PCR using specific primers, and the amplified fragments were inserted between the EcoRI and BamHI sites of the pLVX-Tight-Puro vector. The primers that were used to construct the various deletion and site-directed mutants are shown in Table 1.

\section{Cell culture and transfection}

The 293T cell lines were grown in DMEM high-glucose medium supplemented with $10 \%$ fetal bovine serum (FBS; Hyclone, USA), and cultured at $37^{\circ} \mathrm{C}$ in $5 \% \mathrm{CO}_{2}$ in a humidified incubator. To trace localization patterns, 293T cell lines were seeded onto glass coverslips coated with poly-L-lysine (Sigma-Aldrich, USA) on 6-well plates. Cells were grown overnight and transfected with $1 \mu \mathrm{g}$ GFPfusion protein plasmids expressing Siva and Siva mutants using Lipofectamine ${ }^{\mathbb{B}} 2000$ reagent (Invitrogen, USA) in accordance with the manufacturer's protocol. The original medium was replaced the following day with DMEM supplemented with $5 \%$ FBS, $1 \mu \mathrm{g} / \mathrm{mL}$ tetracycline, and $100 \mathrm{U} / \mathrm{mL}$ penicillin/streptomycin to induce protein expression.

\section{Microscopy}

Prior to imaging, cells were fixed with $4 \%$ formaldehyde buffered in PBS for 30 min at $37^{\circ} \mathrm{C}$ and washed three times with PBS. Nuclei were stained with DAPI $(1 \mu \mathrm{g} / \mathrm{mL})$. The coverslips were mounted on glass slides. The images in Figures 1, 3, and 4 were viewed using a Leica epifluorescence microscope (DMI6000B; Leica, Germany). The images in Figure 2 were acquired with a confocal microscope (LSM710; Zeiss, Germany) at $100 \times$. GFP was imaged using the solid phase argon laser at $488 \mathrm{~nm}$ and a 525/50-band pass filter.

\section{Results}

\section{$\mathrm{N}$-terminal was essential for Siva-1 nuclear localization}

To determine which domain was involved in Siva-1 nuclear localization, we made a series of fusion proteins at the $\mathrm{C}$-terminus of GFP with the various domain deletions (Figure 1A). After transfecting these fusion proteins into 293T cells, we observed their subcellular localization using fluorescence microscopy. Five domain deletion mutants $\triangle \mathrm{SAH}, \triangle \mathrm{DDHR}, \Delta \mathrm{B}-\mathrm{BOX}, \Delta$ zinc finger, and $\Delta \mathrm{C}$-terminal were found to be predominantly expressed in the nucleus, as was wild-type Siva-1 (Figure 1B). However, the $\mathrm{N}$-terminal domain deletion mutant $\Delta 1-33$ was not observed in the nucleus (Figure 1B). These results suggest that the $\mathrm{N}$-terminal 1-33 region plays an essential role in Siva nuclear localization and that the other domains are less involved.

\section{$\mathrm{N}$-terminal AA residues 1-33 were sufficient for nuclear localization}

As shown in Figure 1, we designed mutants encompassing only the Siva-1 1-33 region (Figure 2A), fused them to the C-terminus of GFP, and expressed the fusion proteins in 293T cells. In the presence of $\mathrm{N}$-terminal AAs 1-33, GFP fluorescence was enhanced in the nucleus, displaying a distribution pattern similar to that of wild-type Siva-1 (Figure 2B). This altered distribution of GFP indicated that these $A A$ residues were sufficient for nuclear transport.

\section{$\mathrm{N}$-terminal AA residues 1-18 were crucial but insufficient for Siva-1 localization into the nucleus}

To identify the AAs that participate in nuclear localization of Siva-1, we mutated N-terminal AAs 1-33 in five fusion proteins, replacing each AA with phenylalanine in each mutant (Figure 3A). In the 293T cells, we observed that changes in AAs 13-18 were accompanied by a change in subcellular localization compared with that observed with wild-type Siva-1 (Figure 3B), and mutations of $A A$ residues 1-6 and $7-12$ of the 33 $\mathrm{N}$-terminal AAs of Siva-1 resulted a slightly enhanced presence in the cytoplasm. Other mutants showed no alterations in localization distribution (Figure 3B). These results indicated that $\mathrm{N}$-terminal $\mathrm{AAs}$ 1-18 were crucial to Siva-1 nuclear localization. To determine whether 
Table 1. Primer sequences used for Siva-1 mutations.

\begin{tabular}{|c|c|}
\hline Name of construct & Primer sequence $\left(5^{\prime}-3^{\prime}\right)$ \\
\hline \multirow[t]{2}{*}{ Siva1-18 } & F: AATAAGGATCCATGCCCAAGCGGAGCTGC \\
\hline & R: AATAGAATTCTCAGACTTTGAGTTGGAGCGG \\
\hline \multirow[t]{2}{*}{ Siva1-33 } & F: AATAAGGATCCGCTGCTGAAGGCTGTGTCTG \\
\hline & R: AATAAGAATTCTCAGCGCTCGGCGAACACACC \\
\hline \multirow[t]{2}{*}{ Siva $\Delta 1-33$} & F: AATAAGGATCCATGTACTCACGCGAGGTCTTCG \\
\hline & R: CACTTAGAATTCTCAGGCTTCAAACATAGCACC \\
\hline \multirow{2}{*}{ Siva $\Delta 36-55$} & F: AGCCACGGTGTGTTCGCCGAGCGCTACTCATCACATATCGAGCGAAGATTG \\
\hline & R: TTCACGGAACAATCTTCGCTCGATATGTGATGAGTAGCGCTCGGCGAACA \\
\hline \multirow[t]{2}{*}{$\operatorname{Siva} \Delta 48-114$} & F: GTCTTCGAAAGAACCAAGCAGCTCCTTTTCTGTTCATCGTGCATGAGATC \\
\hline & R: CCCATCCACAGATCTCATGCACGATGAACAGAAAAGGAGCTGCTTGGTTC \\
\hline \multirow[t]{2}{*}{ Siva $\Delta 114-142$} & F: AGGTGGCCTTCCCAGGACAGCGCCCATCGCTTGGGGCTGCGGTGCTTTGG \\
\hline & R: AGCACACAGGCCAAAGCACCGCAGCCCCAAGCGATGGGCGCTGTCCTGGG \\
\hline \multirow[t]{2}{*}{$\operatorname{Siva} \Delta 142-175$} & F: AATAAGGATCCGCTGCTGAAGGCTGTGTCTG \\
\hline & R: CCTCAGAATTCTCAGCCCCAGCAGGTGTATACAC \\
\hline \multirow{2}{*}{ Siva $\Delta 114-175$} & F: AATAAGGATCCGCTGCTGAAGGCTGTGTCTG \\
\hline & R: CCTCAGAATTCTCAAGCGATGGGCGCTGTCCTGG \\
\hline \multirow[t]{2}{*}{ Siva mutant1-6 } & F: TGGACGAGCTGTACAAGATGGCCGCGGCGGCCGCCCCGTTCGCAGACGCAGCCCC \\
\hline & R: GGGGCTGCGTCTGCGAACGGGGCGGCCGCCGCGGCCATCTTGTACAGCTCGTCCA \\
\hline \multirow[t]{2}{*}{ Siva mutant7-12 } & F: TGCCCAAGCGGAGCTGCGCGGCCGCAGCCGCAGCCCCGCTCCAACTCAAAGTCCA \\
\hline & R: TGGACTTTGAGTTGGAGCGGGGCTGCGGCTGCGGCCGCGCAGCTCCGCTTGGGCA \\
\hline \multirow[t]{2}{*}{ Siva mutant13-18 } & F: CGTTCGCAGACGCAGCCGCGGCCGCAGCCGCAGCCCACGTGGGCCTGAAAGAGCT \\
\hline & R: AGCTCTTTCAGGCCCACGTGGGCTGCGGCTGCGGCCGCGGCTGCGTCTGCGAACG \\
\hline \multirow[t]{2}{*}{ Siva mutant19-24 } & F: CGCTCCAACTCAAAGTCGCCGCGGCCGCGGCAGCGCTGAGCCACGGTGTGTTCGC \\
\hline & R: GCGAACACACCGTGGCTCAGCGCTGCCGCGGCCGCGGCGACTTTGAGTTGGAGCG \\
\hline \multirow[t]{2}{*}{ Siva mutant25-33 } & F: GTGGGCCTGAAAGAGGCGGCCGCCGCGGCGGCCGCCGCGGCCTACTCACGCGAGGTC \\
\hline & R: GACCTCGCGTGAGTAGGCCGCGGCGGCCGCCGCGGCGGCCGCCTCTTTCAGGCCCAC \\
\hline \multirow[t]{2}{*}{ Siva(Y34F) } & F: AGCTGAGCCACGGTGTGTTCGCCGAGCGCTTCTCACGCGAGGTCTTCGAAAG \\
\hline & R: TGCTTGGTTCTTTCGAAGACCTCGCGTGAGAAGCGCTCGGCGAACACACC \\
\hline \multirow[t]{2}{*}{ Siva(Y140F) } & F: GCGAGCGGGCCCTGTGTGGGCAGTGTGTATTCACCTGCTGGGGCTGCGGTG \\
\hline & R: CAGGCCAAAGCACCGCAGCCCCAGCAGGTGAATACACACTGCCCACACAG \\
\hline \multirow[t]{2}{*}{ Siva(Y53F) } & F: AGCAGCTCCTTTTCCAAGGGGCTCGGGCCTTTAGAGATCACATATCGAGCG \\
\hline & R: GAACAATCTTCGCTCGATATGTGATCTCTAAAGGCCCGAGCCCCTTGGAA \\
\hline \multirow[t]{2}{*}{ Siva(Y158F) } & F: TGGCCTGTGTGCTGTGTGGCCTTGCAGACTTTGCCGACGATGGTGAGAAGAC \\
\hline & R: GTGCACAGTGTCTTCTCACCATCGTCGGCAAAGTCTGCAAGGCCACACAG \\
\hline
\end{tabular}

these 18 AAs were sufficient for protein translocation into the nucleus, we fused them with the GFP protein and found that in transfected 293T cells there were no differences in the distribution of this fusion protein and that of GFP, which indicated that N-terminal AAs 1-18 are not sufficient for Siva-1 nuclear localization (Figure $3 B$ ). These results suggested that the $\mathrm{N}$-terminal $A A$ residues 1-18 were crucial to but insufficient for Siva-1 nuclear localization.

\section{Siva-1 nuclear import was independent of tyrosine and cysteine sites}

There are four tyrosine residues, Tyr34, Tyr53, Tyr140, and Tyr158, in the Siva-1 protein. To determine whether specific tyrosine residue(s) could affect nuclear localization of Siva-1, we replaced these tyrosines with phenylalanine to produce three mutants involving two residues, Y34F and $\mathrm{Y} 140 \mathrm{~F}, \mathrm{Y} 53 \mathrm{~F}$ and $\mathrm{Y} 158 \mathrm{~F}$, and a quadruple mutant (Figure 4A). All these mutants presented predominantly in the nuclear fraction of 293T cells, as does wild-type Siva-1 (Figure 4B). These results suggested that alteration of tyrosine residues did not prevent Siva-1 nuclear localization.

Cysteine-rich regions located at the C-terminal of Siva-1 include thirteen cysteines. To investigate whether these cysteine residues are involved in nuclear localization of Siva-1, we performed a multiple site-direct mutagenesis substituting all thirteen cysteines with glycine in the 113-170 AA region. After transfection of this mutant into 293T cells, we observed no differences in compartmentalization pattern between mutant and wildtype Siva fusion proteins (Figure 4). This result suggested that these thirteen cysteine residues were not indispensable for Siva-1 nuclear localization. 


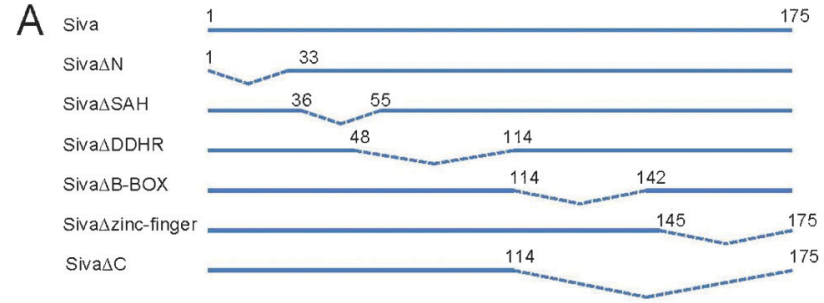

B

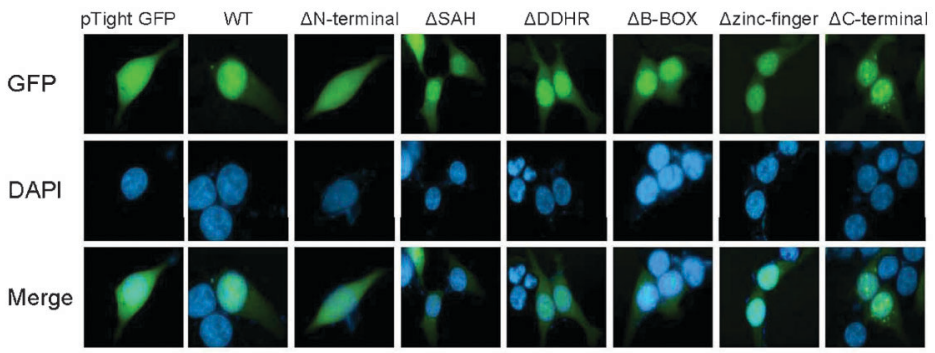

Figure 1. N-terminal is essential for Siva-1 nuclear localization. $A$, Structures of Siva-1 and different deletion mutants of Siva-1. Dotted lines indicate the deleted amino acid sequences within the native molecule. $B$, Subcellular localization of Siva-1 and Siva-1 domain deletion mutants in 293T cells as observed by fluorescence microscopy. GFP: green fluorescent protein; WT: wild type; SAH: Siva amphipathic helical region; DDHR: death domain homology region; B-BOX: B-Box C-H domain.

\section{Discussion}

Most researchers consider Siva-1 to be a cytoplasmic protein even though a few reports have described its presence in the nucleus. A recent study revealed that Siva-1 can interact with transcription factor p53 (11), which indicates that the function of Siva-1 is not limited to apoptosis. Delineating the mechanism by which Siva-1 relocates to the nucleus could help reveal the nuclear function of Siva-1. In this study, we constructed a series of comprehensive domain deletions and site-directed mutants of Siva-1 to determine which domains or specific AAs are required for its nuclear localization. We discovered that the $\mathrm{N}$-terminal 1-33 AA region was required for nuclear localization, but that the SAH, DDHR, B-BOX, and zinc-finger domains, and all four tyrosines and thirteen cysteines were not involved (Figure 1). We also demonstrated that only the $33 \mathrm{~N}$-terminal AA residues of Siva-1 were required for nuclear localization of the protein (Figure 2). Furthermore, we found that $\mathrm{N}$-terminal AAs 1-18 could significantly inhibit Siva-1 entrance into the nucleus (Figure 3 ). These results demonstrated that the $33 \mathrm{~N}$-terminal AAs could act as the NLS, and allow its cargo protein to enter the nucleus. However, Siva-1 Nterminal 1-18 AA region could not cargo GFP into the nucleus, demonstrating a deficit function of NLS in this region (Figure 3 ). It has been shown that USP1 contains three candidate NLSs and those three AA regions display different degrees of nuclear import activity, but USP1 nuclear localization requires any two of the three (13). We hypothesized that Siva-1 nuclear localization may employ a similar mechanism, and include several domains for nuclear localization. If any one of these is absent, another may replace its function in nuclear localization. Thus, mutations in one part of the $\mathrm{N}$-terminal region, i.e., AAs 1$6,7-12,13-18,19-24$, or 25-33 alone could not completely abrogate Siva-1 nuclear localization ability. More studies are needed to clarify the mechanism of Siva-1 N-terminal cargo protein nuclear localization.

NLSs are divided into three groups as follows: 1) Classical nuclear transport, which includes monopartite

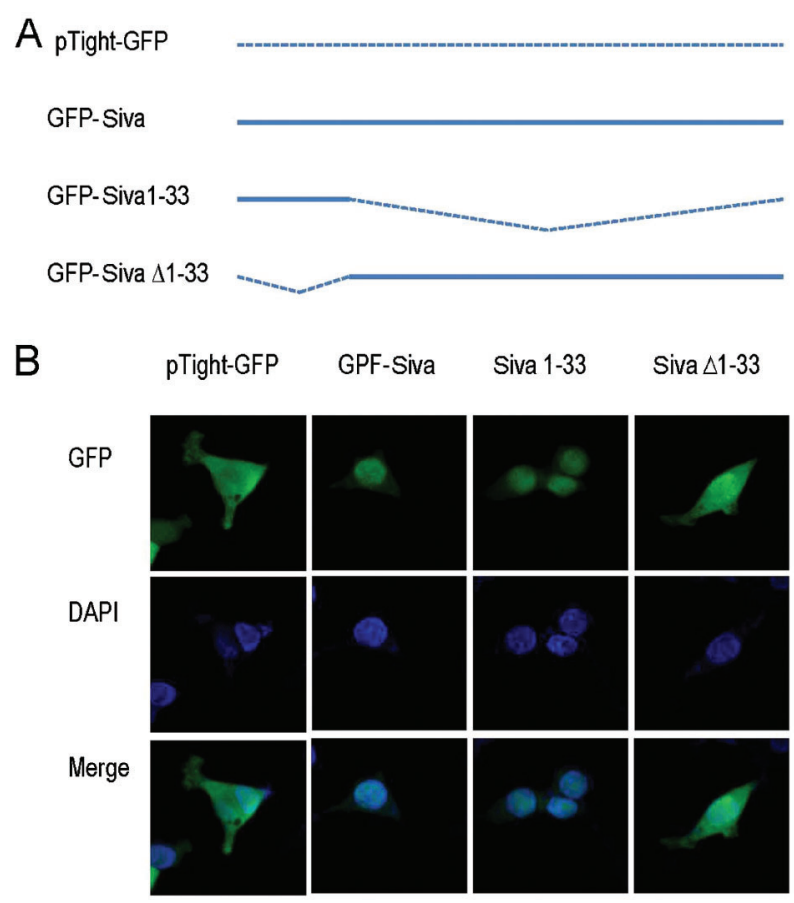

Figure 2. N-terminal amino acid residues 1-33 were sufficient for nuclear localization. Confocal laser scanning microscopy of 293T cells transfected with green fluorescent protein (GFP), GFP-Siva, GFP-Siva1-33, GFP-Siva $\Delta 1-33$, and counter-stained with DAPI to show the location of the nuclear DNA. 


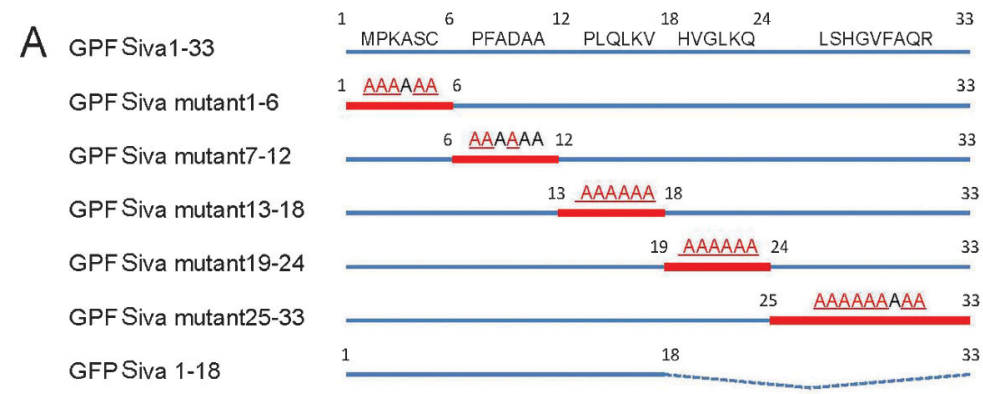

Figure 3. N-terminal amino acid residues 1-18 were necessary but insufficient for Siva-1 localization in the nucleus. 293T cells transfected with green fluorescent protein (GFP)-Siva mutant1-6, GFP-Siva mutant7-12, GFP-Siva mutant13-18, GFP-Siva mutant19-24, GFPSiva mutant25-33, and GFP-Siva1-18. Cells counter-stained with DAPI to show the location of the nuclear DNA.

B

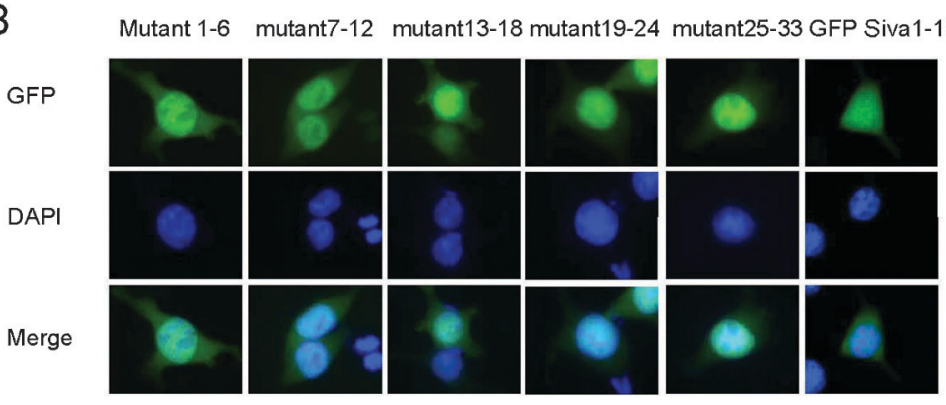

and bipartite signals. The monopartite NLS prototype is the SV40 large T antigen (126PKKKRRV132), characterized by one short basic stretch containing 4-8 lysine and arginine residues, and the bipartite NLS has an NLS motif (155KRPAATKKAGQAKKKK170) that was originally in the Xenopus protein nucleoplasmin (14). It is composed of two peptide regions containing basic residues separated by a mutation-tolerant linker region of 10-12 AAs. 2) Proline-tyrosine-NLS (PY-NLS), characterized by a proline-tyrosine $A A$ domain in the $\mathrm{N}$-terminal, which allows the protein to bind to importin $\beta 2$ (also known as transportin or karyopherin $\beta 2$ ), and then relocates the cargo protein into the nucleus (15). 3) Other types of NLS that are not as common as the classical basic-type NLS and do not contain basic AA residues. By analyzing the $\mathrm{N}$-terminal sequence of murine Siva-1, no consensus sequence was found with the classical NLS and PY-NLS. Therefore, one possible mechanism underlying the transportation of Siva-1 into the nucleus could function by an unknown non-typical NLS located within the

\section{A Siva(Y34F, Y140F) \\ Siva(Y53F, Y158F) \\ Siva(Y34F, Y53F, Y1 40F, Y158F) \\ Sivacystine mutant}

$\mathrm{B}$
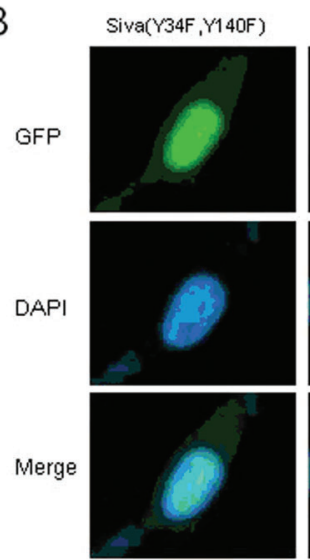

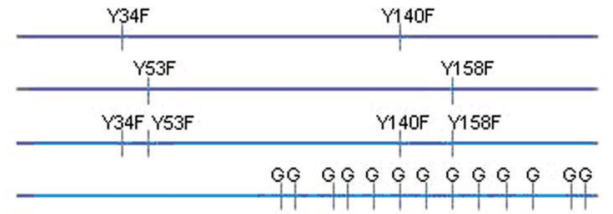

Siva(Y53F, Y158F) Siva(Y34F, Y53F, Y1 40F, Y158F) Siva cystine mutant
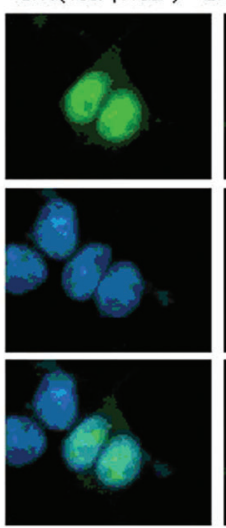
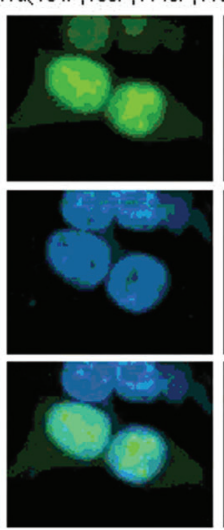
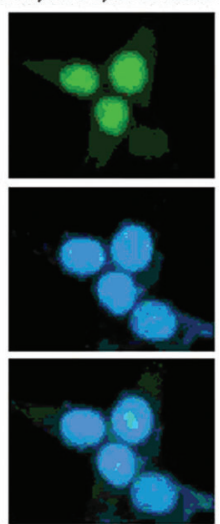

Figure 4. Role of tyrosine and cysteine substitution in the subcellular distribution of Siva-1. Y34F and Y140F, Y53F and Y158F, quadruple mutant, and cysteine mutant were transfected into 293T cells and nuclei were counterstained with DAPI (blue). GFP: green fluorescent protein. 
$\mathrm{N}$-terminal region of Siva-1. It is also possible that Siva-1 does not include an NLS domain, but rather enters the nucleus through its interaction partner protein. Some reports have described several proteins that interact with the Siva-1 N-terminal region. One is p53, a tumor suppressor that is associated with the Siva-1 N-terminal or DDHR domains, which promote protein degradation and inhibition of its activity (11). The interaction sites of XIAP were mapped to the $\mathrm{N}$-terminal, SAH and DDHR domains of Siva-1 (12). HPV-16 E7 binds to the 1-36 AA region of Siva-1 (16). Peroxisomal membrane protein pmp2 binds to the 53-61 AA region of Siva-1 (4). Pyrin is associated with Siva-1 N-terminal domain (6). The Nterminal 1-36 AAs of Siva-1 are required for the connection between Siva-1 and Tyk2. SLIMMER, a nuclear protein, also binds to Siva-1, although it remains unclear which domain is required (17). Among these proteins, p53, SLIMMER, Tyk2, and XIAP are found both in the nucleus and in the cytoplasm, so they all have the potential to deliver Siva-1 into the nucleus. However, which kind of NLS model Siva-1 employs is yet to be determined.

Cells regulate nuclear protein transport at multiple levels through a number of mechanisms. One of these, phosphorylation/dephosphorylation is a well-known modulator of nuclear protein transportation (18). Entry of the Ewing sarcoma proto-oncoprotein into the nucleus is dependent on phosphorylation of tyrosine 565 in the cterminal NLS (19). Previous studies have demonstrated that tyrosine kinases Tyk2 and ARG can interact with and phosphorylate tyrosines of Siva-1 and induce apoptosis $(5,20)$. To determine whether phosphorylated tyrosine can influence Siva-1 nuclear localization, we mutated all its tyrosine residues and then observed the changes in subcellular distribution of the GFP-Siva-1 fusion protein. We found no significant changes in this distribution

\section{References}

1. Yoon $Y$, Ao Z, Cheng $Y$, Schlossman SF, Prasad KV. Murine Siva-1 and Siva-2, alternate splice forms of the mouse Siva gene, both bind to CD27 but differentially transduce apoptosis. Oncogene 1999; 18: 7174-7179, doi: 10.1038/sj.onc. 1203144 .

2. Py B, Slomianny C, Auberger P, Petit PX, Benichou S. Siva1 and an alternative splice form lacking the death domain, Siva-2, similarly induce apoptosis in T lymphocytes via a caspase-dependent mitochondrial pathway. J Immunol 2004; 172: 4008-4017.

3. Prasad KV, Ao Z, Yoon Y, Wu MX, Rizk M, Jacquot S, et al. CD27, a member of the tumor necrosis factor receptor family, induces apoptosis and binds to Siva, a proapoptotic protein. Proc Natl Acad Sci U S A 1997; 94: 6346-6351, doi: 10.1073/pnas.94.12.6346.

4. Nestler M, Martin U, Hortschansky P, Saluz HP, Henke A, Munder $\mathrm{T}$. The zinc containing pro-apoptotic protein siva interacts with the peroxisomal membrane protein pmp22. pattern, which indicated that tyrosine phosphorylation was not involved in the relocation of Siva-1 to the nucleus (Figure 4).

A previous study has shown that Siva-1 negatively regulates NF- $\mathrm{KB}$ activity in TCR-mediated, activationinduced cell death with implications for peripheral tolerance, T-cell homeostasis and cancer (21). Recently, another report in line with the above result found that Siva-1 could balance NF-кB and JNK signaling to promote apoptosis by physically interacting with XIAP and TAK/TAB1 to form a complex. The interaction site of Siva-1 with XIAP was mapped to a region spanning the $\mathrm{N}$-terminal, SAH and DDHR regions. XIAP can augment p65-NF- $\kappa B$ nuclear translocation (22), and the E3 ubiquitin ligase function of the RING domain of XIAP is required for the activation of NF- $\mathrm{BB}(23)$. Therefore, the $\mathrm{N}$ terminal region of Siva-1 is indispensable for this protein nuclear localization, which is required for attenuation of $N F-\kappa B$ activity. Lack of N-terminal AA 1-33 would block Siva-1 entering the nucleus and disrupt the XIAP-

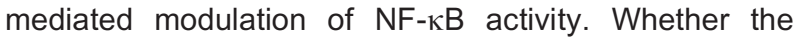
Siva-1 N-terminal AA 1-33 sequence is also essential for regulating NF- $\mathrm{B}$ activity needs future study.

In summary, Siva-1 N-terminal AAs 1-33 were required and sufficient for nuclear localization. Other regions of Siva-1, including SAH, DDHR, B-BOX, zincfinger-like domains, and all tyrosine and cysteine residues, had smaller effects on nuclear localization. Further study is needed to clarify the mechanism and function of Siva-1 in the nucleus.

\section{Acknowledgments}

Research supported by the Natural Science Foundation of China (\#31170831), and the Guangdong Innovative R\&D Team Program (\#2009010058).
Mol Cell Biochem 2006; 287: 147-155, doi: 10.1007/s11010005-9082-6.

5. Shimoda HK, Shide K, Kameda T, Matsunaga T, Shimoda $\mathrm{K}$. Tyrosine kinase 2 interacts with the proapoptotic protein Siva-1 and augments its apoptotic functions. Biochem Biophys Res Commun 2010; 400: 252-257, doi: 10.1016/ j.bbrc.2010.08.051.

6. Balci-Peynircioglu B, Waite AL, $\mathrm{Hu} \mathrm{C}$, Richards N, Staubach-Grosse A, Yilmaz E, et al. Pyrin, product of the MEFV locus, interacts with the proapoptotic protein, Siva. J Cell Physiol 2008; 216: 595-602, doi: 10.1002/jcp.21435.

7. Lin FT, Lai YJ, Makarova N, Tigyi G, Lin WC. The lysophosphatidic acid 2 receptor mediates down-regulation of Siva-1 to promote cell survival. J Biol Chem 2007; 282 37759-37769, doi: 10.1074/jbc.M705025200.

8. Henke A, Launhardt $\mathrm{H}$, Klement K, Stelzner A, Zell R, Munder T. Apoptosis in coxsackievirus B3-caused diseases: interaction between the capsid protein VP2 and the 
proapoptotic protein siva. J Virol 2000; 74: 4284-4290, doi: 10.1128/JVI.74.9.4284-4290.2000.

9. Py B, Bouchet J, Jacquot G, Sol-Foulon N, Basmaciogullari $\mathrm{S}$, Schwartz O, et al. The Siva protein is a novel intracellular ligand of the CD4 receptor that promotes HIV-1 envelopeinduced apoptosis in T-lymphoid cells. Apoptosis 2007; 12: 1879-1892, doi: 10.1007/s10495-007-0106-4.

10. Chu F, Borthakur A, Sun X, Barkinge J, Gudi R, Hawkins S, et al. The Siva-1 putative amphipathic helical region (SAH) is sufficient to bind to BCL-XL and sensitize cells to UV radiation induced apoptosis. Apoptosis 2004; 9: 83-95, doi: 10.1023/B:APPT.0000012125.01799.4c.

11. Du W, Jiang $P$, Li N, Mei $Y$, Wang $X$, Wen $L$, et al. Suppression of p53 activity by Siva1. Cell Death Differ 2009; 16: 1493-1504, doi: 10.1038/cdd.2009.89.

12. Resch U, Schichl YM, Winsauer G, Gudi R, Prasad K, de Martin R. Siva1 is a XIAP-interacting protein that balances NFkappaB and JNK signalling to promote apoptosis. J Cell Sci 2009; 122: 2651-2661, doi: 10.1242/jcs.049940.

13. Garcia-Santisteban I, Zorroza K, Rodriguez JA. Two nuclear localization signals in USP1 mediate nuclear import of the USP1/UAF1 complex. PLoS One 2012; 7: e38570, doi: 10. 1371/journal.pone.0038570.

14. Hicks GR, Raikhel NV. Protein import into the nucleus: an integrated view. Annu Rev Cell Dev Biol 1995; 11: 155-188, doi: 10.1146/annurev.cb.11.110195.001103.

15. Lee BJ, Cansizoglu AE, Suel KE, Louis TH, Zhang Z, Chook YM. Rules for nuclear localization sequence recognition by karyopherin beta 2. Cell 2006; 126: 543-558, doi: 10.1016/ j.cell.2006.05.049.

16. Severino A, Abbruzzese $C$, Manente $L$, Valderas $A A$, Mattarocci S, Federico A, et al. Human papillomavirus-16 E7 interacts with Siva-1 and modulates apoptosis in $\mathrm{HaCaT}$ human immortalized keratinocytes. J Cell Physiol 2007; 212: 118-125, doi: 10.1002/jcp.21011.

17. Cottle DL, McGrath MJ, Wilding BR, Cowling BS, Kane JM, D'Arcy CE, et al. SLIMMER (FHL1B/KyoT3) interacts with the proapoptotic protein Siva-1 (CD27BP) and delays skeletal myoblast apoptosis. J Biol Chem 2009; 284 26964-26977, doi: 10.1074/jbc.M109.036293.

18. Poon IK, Jans DA. Regulation of nuclear transport: central role in development and transformation? Traffic 2005; 6 : 173-186, doi: 10.1111/j.1600-0854.2005.00268.x.

19. Leemann-Zakaryan RP, Pahlich S, Grossenbacher D, Gehring $\mathrm{H}$. Tyrosine Phosphorylation in the C-Terminal Nuclear Localization and Retention Signal (C-NLS) of the EWS Protein. Sarcoma 2011; 2011: 218483.

20. Cao C, Ren X, Kharbanda S, Koleske AJ, Prasad KV, Kufe D. The ARG tyrosine kinase interacts with Siva-1 in the apoptotic response to oxidative stress. J Biol Chem 2001; 276: 11465-11468, doi: 10.1074/jbc.C100050200.

21. Gudi R, Barkinge J, Hawkins S, Chu F, Manicassamy S, Sun Z, et al. Siva-1 negatively regulates NF-kappaB activity: effect on T-cell receptor-mediated activation-induced cell death (AICD). Oncogene 2006; 25: 3458-3462, doi: 10 1038/sj.onc. 1209381.

22. Hofer-Warbinek R, Schmid JA, Stehlik C, Binder BR, Lipp J De Martin R. Activation of NF-kappa B by XIAP, the $X$ chromosome-linked inhibitor of apoptosis, in endothelial cells involves TAK1. J Biol Chem 2000; 275: 22064-22068, doi: 10.1074/jbc.M910346199.

23. Lewis J, Burstein E, Reffey SB, Bratton SB, Roberts AB, Duckett CS. Uncoupling of the signaling and caspaseinhibitory properties of X-linked inhibitor of apoptosis. J Biol Chem 2004; 279: 9023-9029, doi: 10.1074/jbc.M31289 1200. 